\title{
Screening for Hilar Biliary Invasion in Ampullary Cancer Patients
}

\section{Mitsuru Sugimoto ( $\nabla$ kita335@fmu.ac.jp )}

Fukushima Medical University

\section{Tadayuki Takagi}

Fukushima Medical University

Rei Suzuki

Fukushima Medical University

\section{Naoki Konno}

Fukushima Medical University

\section{Yuki Sato}

Fukushima Medical University

Hiroki Irie

Fukushima Medical University

\section{Yoshinori Okubo}

Fukushima Medical University

Jun Nakamura

Fukushima Medical University

Mika Takasumi

Fukushima Medical University

Minami Hashimoto

Fukushima Medical University

\section{Tsunetaka Kato}

Fukushima Medical University

\section{Ryoichiro Kobashi}

Fukushima Medical University

\section{Takumi Yanagita}

Fukushima Medical University

\section{Yuko Hashimoto}

Fukushima Medical University

\section{Shigeru Marubashi}

Fukushima Medical University

\section{Takuto Hikichi}

Fukushima Medical University Hospital 


\section{Research Article}

Keywords: ampullary cancer, biliary biopsy, contrast-enhanced CT, hilar biliary invasion, intraductal ultrasonography

Posted Date: August 10th, 2021

DOl: https://doi.org/10.21203/rs.3.rs-752533/v1

License: (c) (1) This work is licensed under a Creative Commons Attribution 4.0 International License. Read Full License 


\section{Abstract}

Background: The treatment for ampullary cancer is pancreatoduodenectomy or local ampullectomy. However, effective methods for the preoperative investigation of hilar biliary invasion in ampullary cancer patients have not yet been identified. In this study, we aimed to determine an appropriate method for investigating hilar biliary invasion of ampullary cancer.

Methods: Among 43 ampullary cancer patients, 34 underwent endoscopic treatment $(n=9)$ or surgery $(n$ $=25$ ). Imaging findings (thickening and enhancement of the bile duct wall on contrast-enhanced CT (CECT), irregularity on endoscopic retrograde cholangiography (ERC), thickening of the entire bile duct wall on intraductal ultrasonography (IDUS), and partial thickening of the bile duct wall on IDUS) and biliary biopsy results were compared with respect to their ability to diagnose hilar biliary invasion of ampullary cancer.

Results: Hilar invasion was not observed in every patient. Among the patients who did not undergo biliary stent insertion, the combination of partial thickening of the bile duct wall on IDUS and biliary biopsy showed the highest accuracy (100\%) for diagnosing biliary invasion. However, each imaging finding and biliary biopsy yielded some false positive results.

Conclusions: Although some false positive results were obtained with each method, the combination of partial thickening of the bile duct wall on IDUS and biliary biopsy was useful for diagnosing hilar biliary invasion of ampullary cancer. However, hilar invasion of ampullary cancer is rare; therefore, the investigation of hilar biliary invasion of ampullary cancer might be unnecessary.

Trial registration: not applicable

\section{Background}

The standard treatment for ampullary cancer is pancreatoduodenectomy. In addition, local surgical resection of the ampulla or endoscopic ampullectomy has been recently performed for ampullary cancer that does not invade the sphincter of Oddi [1-6]. To perform these treatments, an accurate assessment of the extent of biliary invasion is important. Although ampullary lesions show ductal invasion [7-9], hilar biliary invasion by ampullary lesions has not been reported. When a tumor advances to the hilar biliary duct, the extent of resection should be modified accordingly.

The efficacy of contrast-enhanced CT (CECT), endoscopic retrograde cholangiography (ERC), and intraductal ultrasonography (IDUS) in diagnosing the horizontal progression of bile duct cancer has been reported [10-15]. The diagnostic accuracy of CECT for lateral extension of hilar biliary cancer is reported to be $71-96 \%[13,14,16-23]$. In addition, ERC following IDUS was reported to be useful for diagnosing the lateral extension of biliary ductal cancer [24-27]. The diagnostic accuracy of mapping biopsy for lateral extension of biliary ductal cancer was reported to be 73.0-89.0\% [28-31]. However, whether these 
methods are effective for investigating hilar invasion in ampullary cancer patients is unknown. In this study, we aimed to reveal the best method for diagnosing hilar invasion in ampullary cancer patients.

\section{Methods}

\section{Study design and ethics}

This retrospective study aimed to identify an appropriate screening method for hilar biliary invasion of ampullary cancer. This study was approved by the Institutional Review Board of Fukushima Medical University (approval number: 2453).

\section{Patients}

This study enrolled 43 ampullary cancer patients who were treated at Fukushima Medical University between September 2009 and December 2020. Among them, 34 patients underwent resection by endoscopic treatment $(n=9)$ or surgery $(n=25)$ (Table 1). Endoscopic ampullectomy was performed when invasion into the muscular layer or bile and pancreatic ducts was not observed by ERC or IDUS. It was not necessary to obtain informed consent from the patients because this study was retrospective in design and used previously anonymized clinical data. All patients agreed to receive the clinical examination and treatment by providing written consent; in the case of participants under 18 years of age, consent was obtained from a parent and/or legal guardian. The details of the study can be found on the homepage of Fukushima Medical University. All methods were carried out in accordance with relevant guidelines and regulations. 
Table 1

Patient characteristics and treatment.

\begin{tabular}{|ll|}
\hline Total patients, $\mathrm{n}$ & $\mathbf{4 3}$ \\
\hline Unresectable or treated in other hospitals, $\mathrm{n}$ & 9 \\
\hline Underwent resection, $\mathrm{n}$ & 34 \\
\hline Age, years (mean \pm standard deviation) & $68.0 \pm 11.1$ \\
\hline Sex, $\mathrm{n}$ (male/female) & $20 / 14$ \\
\hline UICC stage 8th edition, $\mathrm{n}$ & \\
\hline I & 16 \\
\hline II & 8 \\
\hline III & 10 \\
\hline Patients already having biliary stents, $\mathrm{n}$ & 4 \\
\hline Treatment, $\mathrm{n}$ & \\
\hline Endoscopic ampullectomy & 9 \\
\hline Surgery & 25 \\
\hline Hilar biliary invasion, $\mathrm{n}$ & 0 \\
\hline Local recurrence, $\mathrm{n}$ & 0 \\
\hline UICC, Union for International Cancer Control & \\
\hline
\end{tabular}

\section{Examination items}

Final diagnosis of hilar biliary invasion was determined according to histological diagnosis and nonexistence of local recurrence during follow-up for more than six months. When the horizontal margin of the resected specimen was negative, hilar invasion was considered negative.

Useful methods for diagnosing hilar invasion were investigated in 34 ampullary cancer patients who underwent endoscopic therapy or surgery. The assessed imaging findings of hilar biliary invasion were thickening and enhancement of the bile duct wall on CECT (Fig. 1a), irregularity on ERC (Fig. 1b), thickening of the entire bile duct wall on IDUS (Fig. 1c), and partial thickening of the bile duct wall on IDUS (Fig. 1d). The usefulness of hilar biliary biopsy was also considered. Thickening of the bile duct wall on IDUS was defined as a diameter of the bile duct wall greater than $2 \mathrm{~mm}$.

All imaging findings were evaluated by more than two pancreaticobiliary disease specialists. Endoscopic retrograde cholangiopancreatography (ERCP) was performed as follows. A duodenoscope was inserted after sufficient sedation was achieved with midazolam with the patient in a prone position. When the duodenoscope reached the Vater papilla, biliary cannulation was started. Tumor progression was 
evaluated by ERC, IDUS, and hilar biliary biopsy. It is difficult to observe the whole circumference of the bile duct wall by EUS. Therefore, the evaluation of hilar invasion by EUS was not considered in this study.

JF260V, JF240, and TJF240 duodenoscopes (Olympus, Tokyo, Japan) were used. An MTW ERCP tapered catheter (MTW Endoskopie, Wesel, Germany) and Tandem XL cannula (Boston Scientific Japan, Tokyo, Japan) were used as the ERC catheters. Endo Jaw FB231K (Olympus) or Radial Jaw ${ }^{\text {TM }} 4$ Biopsy Forceps (Boston Scientific Japan) were used for biliary biopsy.

Post-ERC pancreatitis (PEP) and adverse events were diagnosed according to Cotton's criteria [32]. PEP was defined as an elevated serum amylase level more than three times the normal upper limit with abdominal pain for more than 24 hours after ERC. In addition, all PEP patients were confirmed to have peripancreatic inflammation by CECT. The severity of PEP was categorized as follows: mild: extended hospitalization for 2-3 days; moderate: extended hospitalization for 4-10 days; and severe: extended hospitalization for more than 10 days, hemorrhagic pancreatitis, and pseudocysts that required intervention. The severity of bleeding was categorized as follows: mild: clinical evidence of bleeding, hemoglobin decrease $<3 \mathrm{~g} / \mathrm{dl}$, and no need for transfusion; moderate: transfusion (4 units or less) and no angiographic intervention or surgery; and severe: transfusion (5 units or more) or intervention (angiographic or surgical).

\section{Statistical analyses}

The imaging findings and biliary biopsy results were compared with respect to their ability to diagnose hilar invasion of ampullary cancer by Fisher's exact test. The Bonferroni method and Holm method were used to adjust for multiple comparisons. EZR (Saitama Medical Centre, Jichi Medical University, Saitama, Japan) was used for statistical analysis. A $P$ value $<0.05$ was considered indicative of a significant difference.

\section{Results}

\section{Patient characteristics and treatment}

The patient characteristics and treatment results are shown in Table 1. The mean age of the patients was $68.0 \pm 11.1$ years. There were 20 male patients and 14 female patients. The numbers of the different lesion stages were as follows: I: 16; II: 8; and III: 10 . Stage was classified according to the Union for International Cancer Control classification 8th edition [33]. Four patients had already undergone biliary stent insertion in other hospitals. No histological hilar biliary invasion or local recurrence was observed in any patient.

\section{Imaging findings and biopsy results of all patients}

Among the methods explored for diagnosing hilar biliary invasion of ampullary cancer, hilar biliary irregularity on ERC showed the highest diagnostic accuracy (thickening and enhancement of the bile duct wall on CECT: $53.1 \%$ (17/32); irregularity on ERC: $89.7 \%$ (26/29); thickening of the entire bile duct wall on 
IDUS: $87.5 \%$ (21/24); partial thickening of the bile duct wall on IDUS $87.5 \%(21 / 24)$, biliary biopsy $72.7 \%$ (8/11), $P$ value $<0.01)$ (Fig. 2a). The diagnostic accuracy of irregularity on ERC for hilar invasion of ampullary cancer was significantly higher than that of thickening and enhancement of the bile duct wall on CECT $(P$ value $=0.02)$.

Comparison among the various combinations (imaging findings and biliary biopsy) for diagnosing hilar biliary invasion revealed that the diagnostic accuracy of irregularity on ERC + biliary biopsy $(96.7 \%$ $(29 / 30))$, thickening of the entire bile duct wall on IDUS + biliary biopsy $(95.8 \%(23 / 24))$, and partial thickening of the bile duct wall on IDUS + biliary biopsy $(95.8 \%(23 / 24))$ were significantly higher than that of thickening and enhancement of the bile duct wall on CECT + biliary biopsy $(62.5 \%(20 / 32), P$ value $<0.01,=0.02$, and $=0.02$, respectively) (Fig. $2 b$ ).

\section{Imaging findings and biopsy of patients who had not received biliary duct stents}

Partial thickening of the bile duct wall on IDUS showed the highest diagnostic accuracy among the explored methods (thickening and enhancement of the bile duct wall on CECT: $57.1 \%$ (16/28); irregularity on ERC: $88.0 \%$ (22/25); thickening of the entire bile duct wall on IDUS: $84.2 \%$ (16/19); partial thickening of the bile duct wall on IDUS 89.5\% (17/19); biliary biopsy: $66.7 \%$ (6/9); $P$ value $<0.035$ but no significant differences in pairwise comparisons) (Fig. 3a).

Among the investigated combinations (imaging findings and biliary biopsy) for diagnosing hilar biliary invasion of ampullary cancer, the combination of partial thickening of the bile duct on IDUS and biliary biopsy showed the highest diagnostic accuracy (thickening and enhancement of the bile duct wall on CECT + hilar biliary biopsy: $64.3 \%$ (18/28); irregularity on ERC + biliary biopsy: $96.2 \%(25 / 26)$; thickening of the entire bile duct wall on IDUS + biliary biopsy: $95.0 \%$ (19/20); partial thickening of the bile duct wall on IDUS + biliary biopsy: $100 \%(20 / 20)$; $P$ value $<0.01)$ (Fig. 3b). The combination of irregularity on ERC and biliary biopsy and the combination of partial thickening of the bile duct wall on IDUS and biliary biopsy each had a significantly higher diagnostic accuracy for hilar biliary invasion of ampullary cancer than the combination of thickening and enhancement of the bile duct wall on CECT and biliary biopsy ( $P$ value $=0.027,0.017)$.

\section{Adverse events}

The adverse events are listed in Table 2. Post-endoscopic ampullectomy bleeding occurred in two patients. Both patients improved with endoscopic hemostasis and transfusion. PEP occurred in three patients, all of whom improved with conservative treatment. 
Table 2

Adverse events of treatment.

\begin{tabular}{|lr|}
\hline Post-endoscopic ampullectomy bleeding, $\mathbf{n}$ & \\
\hline Mild & 0 \\
\hline Moderate & 2 \\
Severe & 0 \\
\hline Post-ERC pancreatitis, $\mathrm{n}$ & \\
\hline Mild & 0 \\
\hline Moderate & 3 \\
\hline Severe & 0 \\
\hline ERC, endoscopic retrograde cholangiography & \\
\hline
\end{tabular}

\section{Discussion}

In this study, we investigated appropriate methods for diagnosing hilar biliary invasion of ampullary cancer. Hilar biliary invasion was not observed in all ampullary cancer patients. Although some false positive results were obtained with each method, the diagnostic accuracy of the combination of partial thickening of the bile duct wall on IDUS and hilar biliary biopsy for hilar biliary invasion was $100 \%$ for patients without biliary stents. On the other hand, thickening and enhancement of the hilar bile duct wall on CECT was not effective for diagnosing this condition.

Ampullary cancer occasionally develops concurrently with upstream biliary ductal cancer [34, 35]. However, as described in the introduction, hilar biliary invasion of resectable ampullary cancer has rarely been reported. In fact, hilar invasion of ampullary cancer was not observed in this study. In past reports that have described the results of treatment or surgery for ampullary cancer, pancreaticobiliary type, lymph node metastasis, advanced T stage, and large tumors were identified as risk factors for poor prognosis [36-41]. Hilar biliary invasion was not listed as a risk factor in these reports. It is possible that investigation of hilar biliary invasion in ampullary cancer is not necessary.

Thickening of the bile duct wall on CECT has been reported in cholestasis caused by several diseases (for example, cholangitis, common bile duct stones, pancreatitis and malignant biliary stricture) [42]. In a past systematic review and meta-analysis, the diagnostic accuracy of CT in assessing the extent of bile duct invasion was 64-96\% [13]. In this study, the diagnostic accuracy of CECT in assessing hilar biliary invasion of ampullary cancer was lower than that reported in the previous meta-analysis. Regarding the CECT findings of ampullary cancer, papillary bulging and organ invasion have been identified as predictive factors of tumor recurrence or poor survival [43]. However, hilar bile duct wall thickness was not mentioned in the associated study. Thickening and enhancement of the hilar bile duct wall on CECT was 
not useful. It is thought that ampullary cancer exists at the exit of the bile duct and that the tumors more often close the biliary duct than other biliary diseases. This closure leads to the thickening of the hilar bile duct wall; however, in this study, ampullary cancer did not invade the hilar bile duct.

The diagnostic accuracy of IDUS was higher among those patients without biliary stents. Biliary drainage can cause bile duct wall thickening, and IDUS should be performed before biliary drainage. On the cancerous portion of the bile duct wall, the thickening has been reported to be heterogeneous and partially protruded $[24-27,44]$. In this study, partial thickening of the bile duct wall on IDUS showed the best accuracy among the investigated methods for diagnosing hilar invasion of ampullary cancer in patients without a biliary stent. Naitoh et al. [45] reported that bile duct wall thickening in the nonstricture region was unremarkable in bile duct cancer patients. However, false-positive cases (diameter of the hilar bile duct wall from 2-3.3 $\mathrm{mm}$ ) were observed in this study. Therefore, the evaluation of the nonstricture portion on IDUS in patients with ampullary cancer is not believed to be equivalent to that in patients with common bile duct cancer. Therefore, the detection of partial thickening of the bile duct wall should be combined with other methods.

The hilar biliary biopsy results were false positive in three cases. Although this number is low, such results might influence the operative method. Therefore, false positives in hilar biliary biopsy should be avoided. Regarding the reason for these false positives, it is highly likely that the biopsy forceps contact the ampullary cancer. The efficacy of cholangioscopy in diagnosing biliary lesions has been reported [46-56]. However, cholangioscopy has difficulty passing ampullary cancer. To avoid contact of the biopsy forceps with the tumor and to improve the diagnostic accuracy of hilar biliary biopsy for ampullary cancer patients, biliary biopsy with a catheter that introduces biopsy forceps could be useful $[30,31]$. When biliary biopsy with a catheter is unavailable, the combination method of biliary biopsy and IDUS should be considered.

This study has some limitations. First, this is a retrospective study performed at a single institution. A multicenter prospective study is needed to verify the results of this study. Second, a few patients underwent all examinations (CECT, ERC, IDUS, and biliary biopsy). In future studies, a higher number of cases would be desirable. Third, as described above, ampullary cancer patients with hilar biliary invasion were not included in this study. To improve the false-negative rate, a study involving cases of hilar biliary invasion is needed.

\section{Conclusions}

Although false-positive results were obtained with each method, the combination of partial thickening of the bile duct on IDUS and biliary biopsy was useful for diagnosing hilar biliary invasion of ampullary cancer. In addition, it is recommended that hilar biliary biopsy be performed through a catheter to avoid contamination from cancer. However, hilar invasion of ampullary cancer is rare, and hilar investigation might be unnecessary for ampullary cancer patients. 


\section{Abbreviations}

CECT: Contrast-enhanced CT; ERC: Endoscopic retrograde cholangiography; IDUS: Intraductal ultrasonography; UICC: Union for International Cancer Control; ERCP: Endoscopic retrograde cholangiopancreatography; PEP: Post-ERC pancreatitis.

\section{Declarations}

\section{Ethics approval and consent to participate}

All the experiment protocol for involving human data was in accordance with the guidelines of national/international/institutional or Declaration of Helsinki in the manuscript. The study protocol was reviewed and approved by the Institutional Review Board of Fukushima Medical University (Number 2453). The analysis used anonymous clinical data obtained after all the participants agreed to treatment by written consent, so patients were not required to provide informed consent for this study. The details of the study can be found on the homepage of Fukushima Medical University.

\section{Consent for publication}

Not applicable

\section{Availability of data and materials}

The datasets generated and/or analyzed during the current study are available from the corresponding author upon reasonable request.

\section{Competing interests}

The authors declare that they have no competing interests.

\section{Funding}

None.

\section{Authors' contributions}

MS wrote the paper, contributed to study design and performed the research; TT contributed to study design and oversaw the research; RS, NK, HA, YS, HI, YO, JN, MT, MH, TK, RK, TY, TH and SM provided clinical advice; $\mathrm{YH}$ performed pathological diagnosis; and $\mathrm{HO}$ supervised the study and the writing of the paper. All authors have read and approved the final manuscript.

\section{Acknowledgments}

We thank all the staff at the Department of Gastroenterology of Fukushima Medical University, the Department of Endoscopy of Fukushima Medical University Hospital, and the gastroenterology ward of 
Fukushima Medical University Hospital. We also thank American Journal Experts for providing English language editing.

\section{References}

1. Bohnacker S, Soehendra N, Maguchi H, Chung JB, Howell DA. Endoscopic resection of benign tumors of the papilla of vater. Endoscopy. 2006;38:521-5.

2. Goldberg M, Zamir O, Hadary A, Nissan S. Wide local excision as an alternative treatment for periampullary carcinoma. Am J Gastroenterol. 1987;82:1169-71.

3. Han J, Kim MH. Endoscopic papillectomy for adenomas of the major duodenal papilla (with video). Gastrointest Endosc. 2006;63:292-301.

4. Knox RA, Kingston RD. Carcinoma of the ampulla of Vater. Br J Surg. 1986;73:72-3.

5. Sharp KW, Brandes JL. Local resection of tumors of the ampulla of Vater. Am Surg. 1990;56:214-7.

6. Tarazi RY, Hermann RE, Vogt DP, Hoerr SO, Esselstyn CB, Jr., Cooperman AM, et al. Results of surgical treatment of periampullary tumors: a thirty-five-year experience. Surgery. 1986;100:716-23.

7. Irani S, Arai A, Ayub K, Biehl T, Brandabur JJ, Dorer R, et al. Papillectomy for ampullary neoplasm: results of a single referral center over a 10-year period. Gastrointest Endosc. 2009;70:923-32.

8. Klein A, Tutticci N, Bourke MJ. Endoscopic resection of advanced and laterally spreading duodenal papillary tumors. Dig Endosc. 2016;28:121-30.

9. van der Wiel SE, Poley JW, Koch AD, Bruno MJ. Endoscopic resection of advanced ampullary adenomas: a single-center 14-year retrospective cohort study. Surg Endosc. 2019;33:1180-8.

10. Anderson MA, Appalaneni V, Ben-Menachem T, Decker GA, Early DS, Evans JA, et al. The role of endoscopy in the evaluation and treatment of patients with biliary neoplasia. Gastrointest Endosc. 2013;77:167-74.

11. Kawakami H, Kuwatani M, Onodera M, Haba S, Eto K, Ehira N, et al. Endoscopic nasobiliary drainage is the most suitable preoperative biliary drainage method in the management of patients with hilar cholangiocarcinoma. J Gastroenterol. 2011;46:242-8.

12. Kawashima H, Itoh A, Ohno E, Itoh Y, Ebata T, Nagino M, et al. Preoperative endoscopic nasobiliary drainage in 164 consecutive patients with suspected perihilar cholangiocarcinoma: a retrospective study of efficacy and risk factors related to complications. Ann Surg. 2013;257:121-7.

13. Ruys AT, van Beem BE, Engelbrecht MR, Bipat S, Stoker J, Van Gulik TM. Radiological staging in patients with hilar cholangiocarcinoma: a systematic review and meta-analysis. Br J Radiol. 
14. Senda $\mathrm{Y}$, Nishio H, Oda K, Yokoyama Y, Ebata T, Igami T, et al. Value of multidetector row $C T$ in the assessment of longitudinal extension of cholangiocarcinoma: correlation between MDCT and microscopic findings. World J Surg. 2009;33:1459-67.

15. Tamada K, Ushio J, Sugano K. Endoscopic diagnosis of extrahepatic bile duct carcinoma: advances and current limitations. World J Clin Oncol. 2011;2:203-16.

16. Lee HY, Kim SH, Lee JM, Kim SW, Jang JY, Han JK, et al. Preoperative assessment of resectability of hepatic hilar cholangiocarcinoma: combined CT and cholangiography with revised criteria. Radiology. 2006;239:113-21.

17. Cho ES, Park MS, Yu JS, Kim MJ, Kim KW. Biliary ductal involvement of hilar cholangiocarcinoma: multidetector computed tomography versus magnetic resonance cholangiography. J Comput Assist Tomogr. 2007;31:72-8.

18. Endo I, Shimada H, Sugita M, Fujii Y, Morioka D, Takeda K, et al. Role of three-dimensional imaging in operative planning for hilar cholangiocarcinoma. Surgery. 2007;142:666-75.

19. Unno M, Okumoto T, Katayose Y, Rikiyama T, Sato A, Motoi F, et al. Preoperative assessment of hilar cholangiocarcinoma by multidetector row computed tomography. J Hepatobiliary Pancreat Surg. 2007;14:434-40.

20. Watadani T, Akahane M, Yoshikawa T, Ohtomo K. Preoperative assessment of hilar cholangiocarcinoma using multidetector-row CT: correlation with histopathological findings. Radiat Med. 2008;26:402-7.

21. Chen HW, Lai EC, Pan AZ, Chen T, Liao S, Lau WY. Preoperative assessment and staging of hilar cholangiocarcinoma with 16-multidetector computed tomography cholangiography and angiography. Hepatogastroenterology. 2009;56:578-83.

22. Akamatsu N, Sugawara Y, Osada H, Okada T, Itoyama S, Komagome M, et al. Diagnostic accuracy of multidetector-row computed tomography for hilar cholangiocarcinoma. J Gastroenterol Hepatol. 2010;25:731-7.

23. Kim HM, Park JY, Kim KS, Park MS, Kim MJ, Park YN, et al. Intraductal ultrasonography combined with percutaneous transhepatic cholangioscopy for the preoperative evaluation of longitudinal tumor extent in hilar cholangiocarcinoma. J Gastroenterol Hepatol. 2010;25:286-92.

24. Moon SH, Kim MH. The role of endoscopy in the diagnosis of autoimmune pancreatitis. Gastrointest Endosc. 2012;76:645-56. 
25. Tabata T, Kamisawa T, Hara S, Kuruma S, Chiba K, Kuwata G, et al. Differentiating immunoglobulin g4-related sclerosing cholangitis from hilar cholangiocarcinoma. Gut Liver. 2013;7:234-8.

26. Kuwatani M, Kawakami H, Zen $Y$, Kawakubo K, Kudo T, Abe $Y$, et al. Difference from bile duct cancer and relationship between bile duct wall thickness and serum IgG/lgG4 levels in IgG4-related sclerosing cholangitis. Hepatogastroenterology. 2014;61:1852-6.

27. Naitoh I, Zen Y, Nakazawa T, Ando T, Hayashi K, Okumura F, et al. Small bile duct involvement in IgG4related sclerosing cholangitis: liver biopsy and cholangiography correlation. J Gastroenterol. 2011;46:269-76.

28. Ito $\mathrm{K}$, Sakamoto $\mathrm{Y}$, Isayama $\mathrm{H}$, Nakai Y, Watadani T, Tanaka M, et al. The impact of MDCT and endoscopic transpapillary mapping biopsy to predict longitudinal spread of extrahepatic cholangiocarcinoma. J Gastrointest Surg. 2018;22:1528-37.

29. Yao S, Taura K, Okuda Y, Kodama Y, Uza N, Gouda N, et al. Effect of mapping biopsy on surgical management of cholangiocarcinoma. J Surg Oncol. 2018;118:997-1005.

30. Okada H, Uza N, Matsumori T, Matsumoto S, Muramoto Y, Ota S, et al. A novel technique for mapping biopsy of bile duct cancer. Endoscopy. 2020;53:647-51.

31. Hijioka S, Hara K, Mizuno N, Imaoka H, Mekky MA, Nagashio Y, et al. A novel technique for endoscopic transpapillary "mapping biopsy specimens" of superficial intraductal spread of bile duct carcinoma (with videos). Gastrointest Endosc. 2014;79:1020-5.

32. Cotton PB, Lehman G, Vennes J, Geenen JE, Russell RC, Meyers WC, et al. Endoscopic sphincterotomy complications and their management: an attempt at consensus. Gastrointest Endosc. 1991;37:383-93.

33. Brierley JD, Gospodarowicz MK, Wittekind C. TNM-classification of malignant tumours. 8th ed. New Jersey: Wiley-Blackwell; 2017.

34. Nishihara K, Tsuneyoshi M, Shimura H, Yasunami Y. Three synchronous carcinomas of the papilla of Vater, common bile duct and pancreas. Pathol Int. 1994;44:325-32.

35. Hirono S, Tani M, Terasawa H, Kawai M, Ina S, Uchiyama K, et al. A collision tumor composed of cancers of the bile duct and ampulla of Vater-immunohistochemical analysis of a rare entity of double cancer. Hepatogastroenterology. 2008;55:861-4.

36. Miyakawa S, Ishihara S, Horiguchi A, Takada T, Miyazaki M, Nagakawa T. Biliary tract cancer treatment: 5,584 results from the Biliary Tract Cancer Statistics Registry from 1998 to 2004 in Japan. J Hepatobiliary Pancreat Surg. 2009;16:1-7.

37. Park HM, Park SJ, Han SS, Hong SK, Hong EK, Kim SW. Very early recurrence following pancreaticoduodenectomy in patients with ampullary cancer. Medicine (Baltimore). 2019;98:e17711. 
38. Zimmermann C, Wolk S, Aust DE, Meier F, Saeger HD, Ehehalt F, et al. The pathohistological subtype strongly predicts survival in patients with ampullary carcinoma. Sci Rep. 2019;9:12676.

39. Moekotte AL, van Roessel S, Malleo G, Rajak R, Ecker BL, Fontana M, et al. Development and external validation of a prediction model for survival in patients with resected ampullary adenocarcinoma. Eur $\mathrm{J}$ Surg Oncol. 2020;46:1717-26.

40. Vilhordo DW, Gregório C, Valentini DF, Jr., Edelweiss MIA, Uchoa DM, Osvaldt AB. Prognostic factors of long-term survival following radical resection for ampullary carcinoma. J Gastrointest Cancer. 2020; doi:10.1007/s12029-020-00479-9.

41. Nappo G, Galvanin J, Gentile D, Capretti G, Pulvirenti A, Bozzarelli S, et al. Long-term outcomes after pancreatoduodenectomy for ampullary cancer: the influence of the histological subtypes and comparison with the other periampullary neoplasms. Pancreatology. 2021. doi:10.1016/j.pan.2021.03.005.

42. Schulte SJ, Baron RL, Teefey SA, Rohrmann CA, Jr., Freeny PC, Shuman WP, et al. CT of the extrahepatic bile ducts: wall thickness and contrast enhancement in normal and abnormal ducts. AJR Am J Roentgenol. 1990;154:79-85.

43. Yoen H, Kim JH, Hur BY, Ahn SJ, Jeon SK, Choi SY, et al. Prediction of tumor recurrence and poor survival of ampullary adenocarcinoma using preoperative clinical and CT findings. Eur Radiol. 2021;31:2433-43.

44. Kamisawa T, Okazaki K. Role of endoscopic retrograde cholangiography in autoimmune pancreatitis. Pancreatology. 2016;16:798-9.

45. Naitoh I, Nakazawa T, Ohara H, Ando T, Hayashi K, Tanaka H, et al. Endoscopic transpapillary intraductal ultrasonography and biopsy in the diagnosis of IgG4-related sclerosing cholangitis. $\mathrm{J}$ Gastroenterol. 2009;44:1147-55.

46. Ramchandani M, Reddy DN, Gupta R, Lakhtakia S, Tandan M, Darisetty S, et al. Role of single-operator peroral cholangioscopy in the diagnosis of indeterminate biliary lesions: a single-center, prospective study. Gastrointest Endosc. 2011;74:511-9.

47. Siddiqui AA, Mehendiratta V, Jackson W, Loren DE, Kowalski TE, Eloubeidi MA. Identification of cholangiocarcinoma by using the Spyglass Spyscope system for peroral cholangioscopy and biopsy collection. Clin Gastroenterol Hepatol. 2012;10:466-71; quiz e48.

48. Manta R, Frazzoni M, Conigliaro R, Maccio L, Melotti G, Dabizzi E, et al. SpyGlass single-operator peroral cholangioscopy in the evaluation of indeterminate biliary lesions: a single-center, prospective, cohort study. Surg Endosc. 2013;27:1569-72.

49. Nishikawa T, Tsuyuguchi T, Sakai Y, Sugiyama H, Miyazaki M, Yokosuka O. Comparison of the diagnostic accuracy of peroral video-cholangioscopic visual findings and cholangioscopy-guided forceps 
biopsy findings for indeterminate biliary lesions: a prospective study. Gastrointest Endosc. 2013;77:21926.

50. Navaneethan U, Hasan MK, Lourdusamy V, Njei B, Varadarajulu S, Hawes RH. Single-operator cholangioscopy and targeted biopsies in the diagnosis of indeterminate biliary strictures: a systematic review. Gastrointest Endosc. 2015;82:608-14.e2.

51. Tanaka R, Itoi T, Honjo M, Tsuchiya T, Kurihara T, Tsuji S, et al. New digital cholangiopancreatoscopy for diagnosis and therapy of pancreaticobiliary diseases (with videos). J Hepatobiliary Pancreat Sci. 2016;23:220-6.

52. Varadarajulu S, Bang JY, Hasan MK, Navaneethan U, Hawes R, Hebert-Magee S. Improving the diagnostic yield of single-operator cholangioscopy-guided biopsy of indeterminate biliary strictures: ROSE to the rescue? (with video). Gastrointest Endosc. 2016;84:681-7.

53. Figueroa-Marrero A, Chavarria-Herbozo CM, de la Serna Higuera C, Perez-Miranda M. Long-standing indeterminate biliary stricture with iterative negative tissue sampling revealed as cholangiocarcinoma under SpyGlassTM cholangiocoscopy. Rev Esp Enferm Dig. 2017;109:220-1.

54. Lee YN, Moon JH, Choi HJ, Lee TH, Choi MH, Cha SW, et al. Direct peroral cholangioscopy for diagnosis of bile duct lesions using an I-SCAN ultraslim endoscope: a pilot study. Endoscopy. 2017;49:675-81.

55. Pereira P, Peixoto A, Andrade P, Macedo G. Peroral cholangiopancreatoscopy with the SpyGlass(R) system: what do we know 10 years later. J Gastrointestin Liver Dis. 2017;26:165-70.

56. Onoyama T, Takeda Y, Kawata S, Kurumi H, Koda H, Yamashita T, et al. Adequate tissue acquisition rate of peroral cholangioscopy-guided forceps biopsy. Ann Transl Med. 2020;8:1073.

\section{Figures}



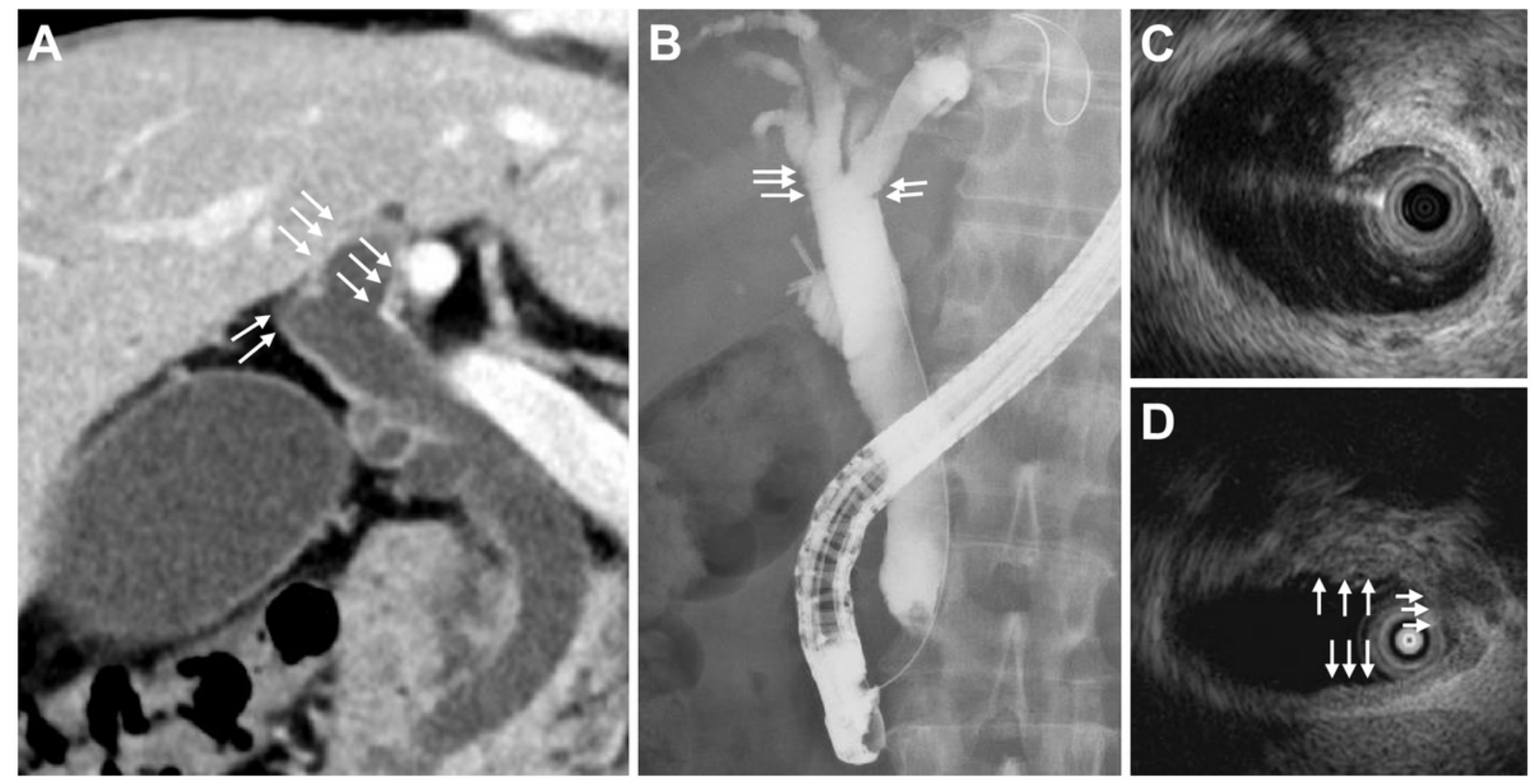

\section{Figure 1}

Imaging findings of the hilar biliary duct. (a) Thickening and enhancement of the bile duct wall on CECT. (b) Irregularity on ERC. (c) Thickening of the entire bile duct wall on IDUS. (d) Partial thickening of the bile duct wall on IDUS. CECT, contrast-enhanced CT; ERC, endoscopic retrograde cholangiography; IDUS, intraductal ultrasonography 


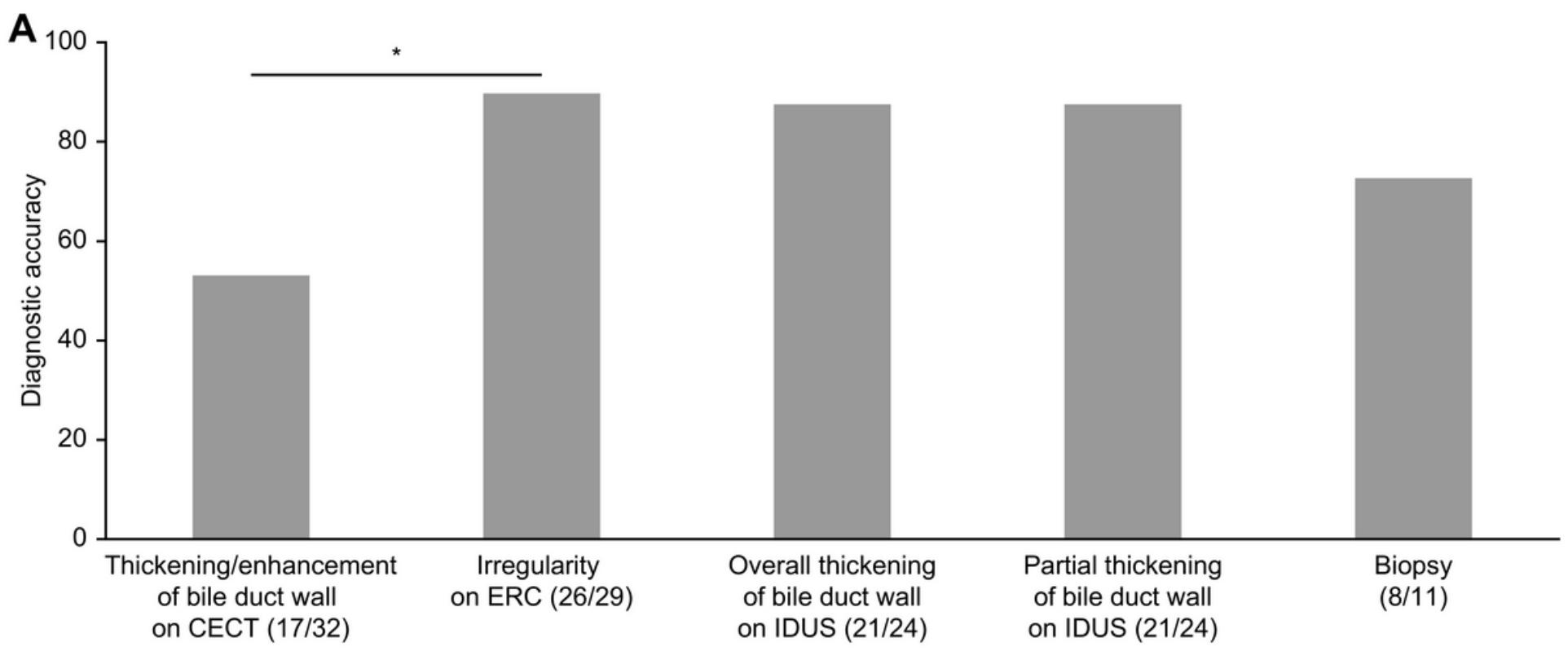

B

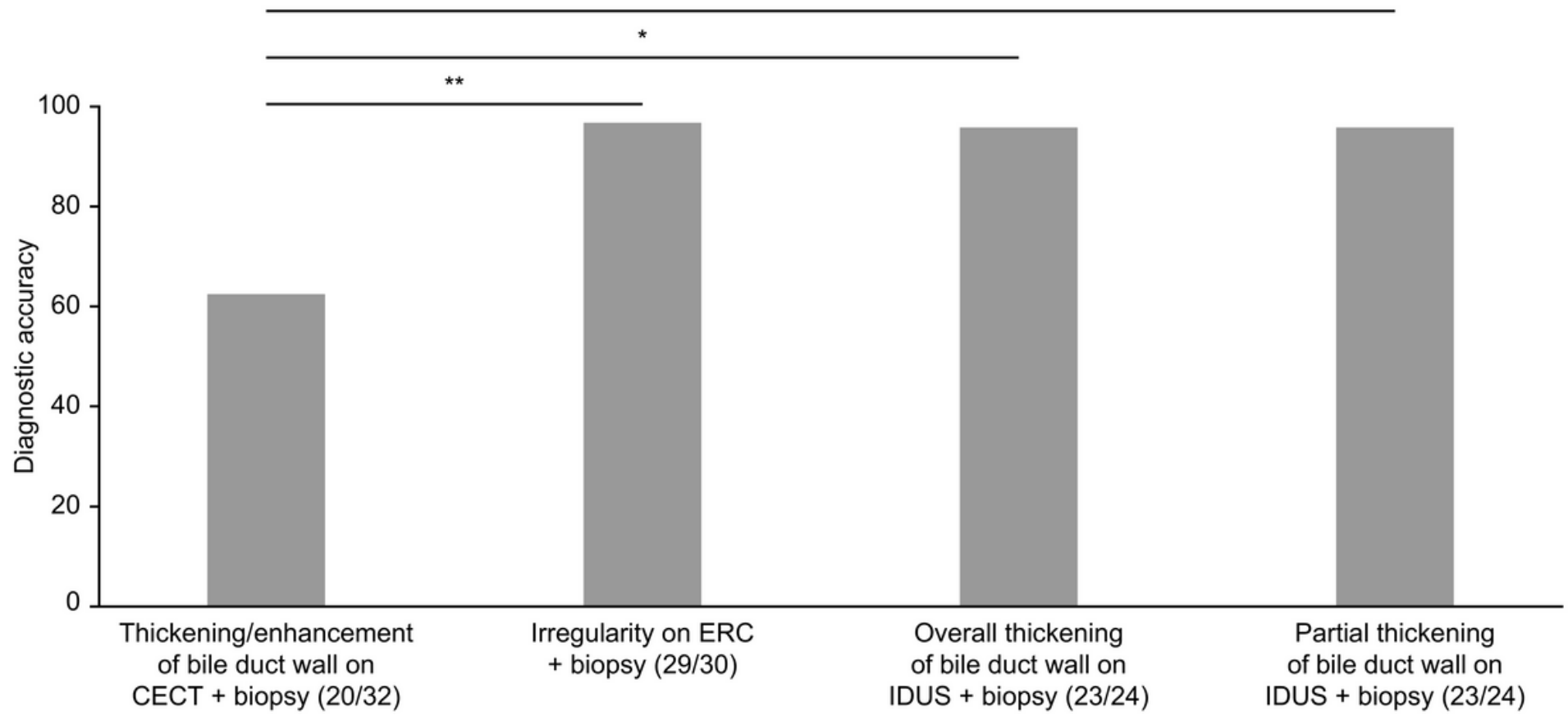

Figure 2

Comparison of methods for diagnosing hilar biliary invasion of ampullary cancer in all patients. (a) Irregularity on ERC showed the highest diagnostic accuracy. (b) Among the various combinations (imaging findings and biliary biopsy) for diagnosing hilar biliary invasion, irregularity on ERC + biliary biopsy showed the highest diagnostic accuracy. ${ }^{\star} P<0.05$, ${ }^{\star *} P<0.01$. ERC, endoscopic retrograde cholangiography. 


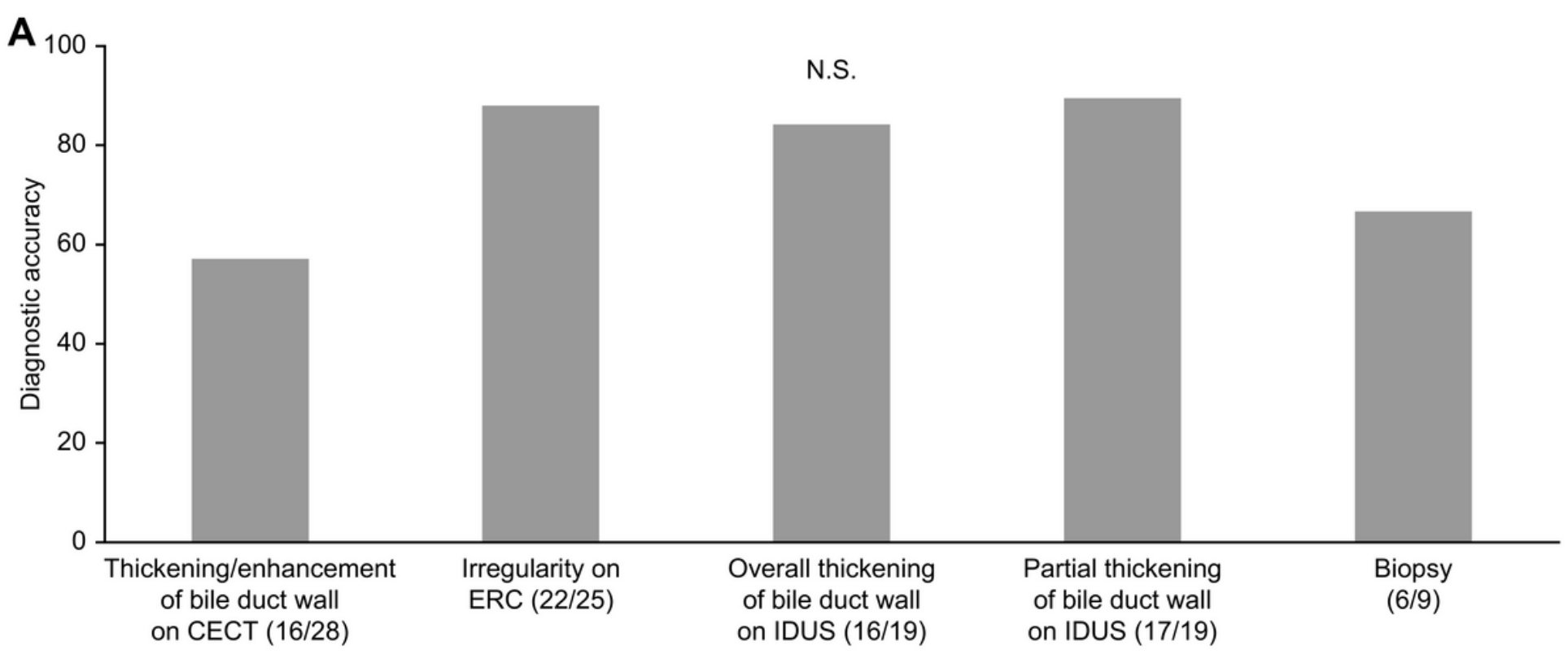

B

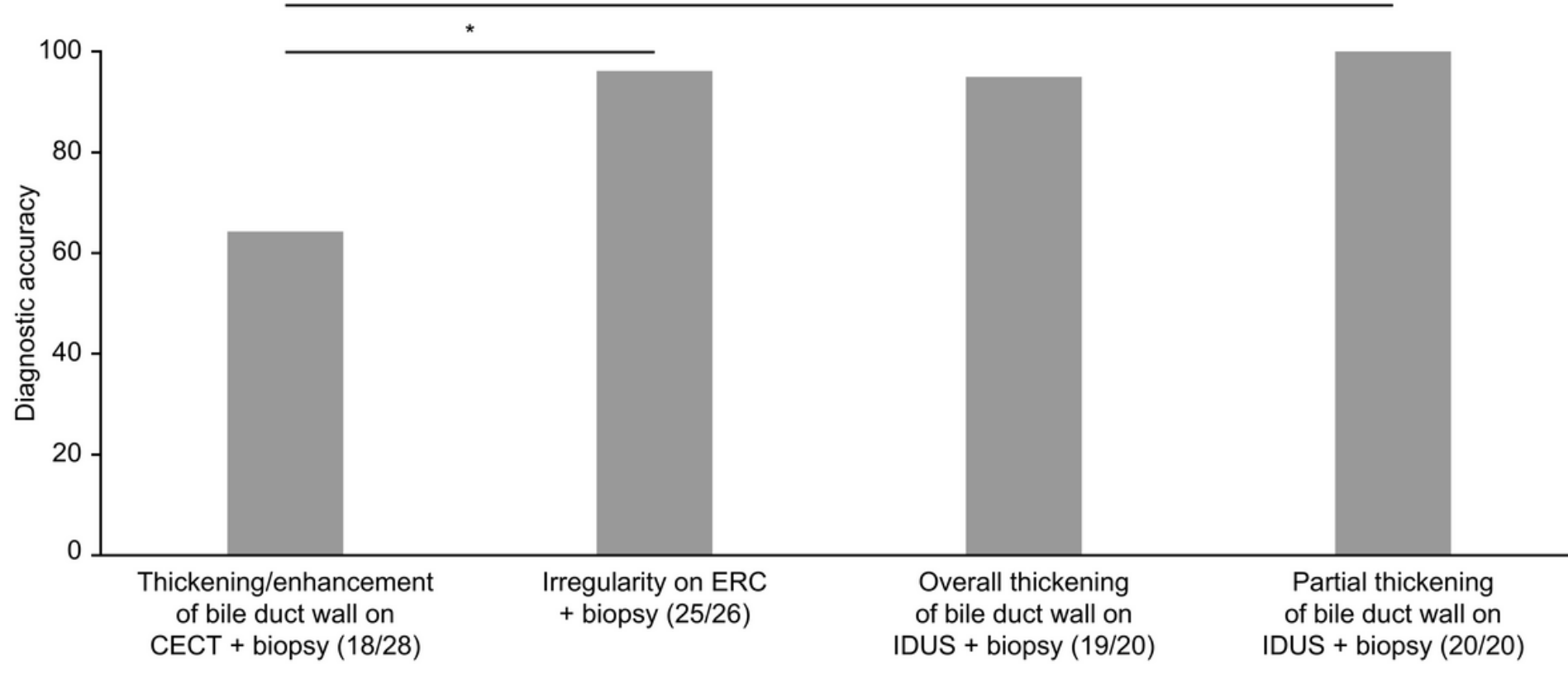

\section{Figure 3}

Comparison of methods for diagnosing hilar biliary invasion of ampullary cancer in patients without biliary stents. (a) Partial thickening of the bile duct wall on IDUS showed the highest diagnostic accuracy. (b) Among the various combinations (imaging findings and biliary biopsy) for diagnosing hilar biliary invasion, partial thickening of the bile duct wall on IDUS + biliary biopsy showed the highest diagnostic accuracy. * $P<0.05$. IDUS, intraductal ultrasonography 